\title{
APPROXIMATELY ADDITIVE MAPPINGS IN NON-ARCHIMEDEAN NORMED SPACES
}

\author{
Alireza Kamel Mirmostafaee
}

\begin{abstract}
We establish a new strategy to study the Hyers-Ulam-Rassias stability of the Cauchy and Jensen equations in non-Archimedean normed spaces. We will also show that under some restrictions, every function which satisfies certain inequalities can be approximated by an additive mapping in non-Archimedean normed spaces. Some applications of our results will be exhibited. In particular, we will see that some results about stability and additive mappings in real normed spaces are not valid in non-Archimedean normed spaces.
\end{abstract}

\section{Introduction}

In 1940, S. M. Ulam [24] in the University of Wisconsin proposed the following question about the stability of homomorphisms:

Let $\left(G_{1}, *\right)$ be a group and $\left(G_{2}, \diamond, d\right)$ be a metric group with the metric $d$. Given $\varepsilon>0$, does there exists a $\delta_{\varepsilon}>0$ such that if a mapping $h: G_{1} \rightarrow G_{2}$ satisfies the inequality

$$
d(h(x * y), h(x) \diamond h(y))<\delta \quad \forall x, y \in G_{1},
$$

then there is a homomorphism $H: G_{1} \rightarrow G_{2}$ with $d(h(x), H(x))<\varepsilon$ for all $x \in G_{1}$ ?

In the next year, D. H. Hyers [8], gave a positive answer to the above question for additive groups under the assumption that the groups are Banach spaces. In 1978, Th. M. Rassias [21] proved a generalization of Hyers' theorem for additive mappings in the following way:

Theorem 1.1. Let $f$ be an approximately additive mapping from a normed vector space $E$ into a Banach space $E^{\prime}$, i.e., $f$ satisfies the inequality

$$
\|f(x+y)-f(x)-f(y)\| \leq \varepsilon\left(\|x\|^{r}+\|y\|^{r}\right)
$$

Received June 19, 2008

2000 Mathematics Subject Classification. Primary 39B82, 39B22, 46S10; Secondary 39B62.

Key words and phrases. Hyers-Ulam-Rassias stability, Cauchy equation, Jensen equation, Jordan-von Neumann-type Jensen inequality. 
for all $x, y \in E$, where $\varepsilon$ and $r$ are constants with $\varepsilon>0$ and $0 \leq r<1$. Then the mapping $L: E \rightarrow E^{\prime}$ defined by $L(x)=\lim _{n \rightarrow \infty} 2^{-n} f\left(2^{n} x\right)$ is the unique additive mapping which satisfies

$$
\|f(x)-L(x)\| \leq \frac{2 \varepsilon}{2-2^{r}}\|x\|^{r}
$$

for all $x \in E$.

The result of Th. M. Rassias has influenced the development of what is now called the Hyers-Ulam-Rassias stability theory for functional equations. In 1994, a generalization of Rassias' theorem was obtained by Găvruta [6] by replacing the bound $\varepsilon\left(\|x\|^{p}+\|y\|^{p}\right)$ by a general control function $\varphi(x, y)$. Several stability results have been recently obtained for various equations, also for mappings with more general domains and ranges (see $[3,9,10,11])$.

A Jensen equation is a equation of the form $2 f\left(\frac{x+y}{2}\right)=f(x)+f(y)$, where $f$ is a mapping between linear spaces. It is easy to see that a mapping $f: X \rightarrow Y$ between linear spaces with $f(0)=0$ satisfies the Jensen equation if and only if it is additive; cf. [20, Theorem 6]. The concept of stability for a functional equation arises when we replace the functional equation by an equality which acts as a perturbation of the equation. Stability of Jensen equation has been studied at first by Kominek [15] and then by several other mathematicians; cf. $[6,12,16,17,18]$.

In 1897, Hensel [7] has introduced a normed space which does not have the Archimedean property. It turned out that non-Archimedean spaces have many nice applications $[4,13,14,19]$.

Definition 1.2. Let $\mathbb{K}$ be a field. A non-Archimedean absolute value on $\mathbb{K}$ is a function ||$: \mathbb{K} \rightarrow \mathbb{R}$ such that for any $a, b \in \mathbb{K}$ we have

(i) $|a| \geq 0$ and equality holds if and only if $a=0$,

(ii) $|a b|=|a||b|$,

(iii) $|a+b| \leq \max \{|a|,|b|\}$.

The condition (iii) is called the strong triangle inequality. By (ii), we have $|1|=|-1|=1$. Thus, by induction, it follows from (iii) that $|n| \leq 1$ for each integer $n$. We always assume in addition that || is non trivial, i.e., that

(iv) there is an $a_{0} \in \mathbb{K}$ such that $\left|a_{0}\right| \neq 0,1$.

Definition 1.3. Let $X$ be a linear space over a scalar field $\mathbb{K}$ with a nonArchimedean non-trivial valuation $|\cdot|$. A function $\|\cdot\|: X \rightarrow \mathbb{R}$ is a nonArchimedean norm (valuation) if it satisfies the following conditions:

(i) $\|x\|=0$ if and only if $x=0$;

(ii) $\|x\|=|r|\|x\|$ for all $r \in \mathbb{K}$ and $x \in X$;

(iii) the strong triangle inequality (ultrametric); namely,

$$
\|x+y\| \leq \max \{\|x\|,\|y\|\} \quad(x, y \in X) .
$$

Then $(X,\|\cdot\|)$ is called a non-Archimedean space. 
By a complete non-Archimedean space we mean one in which every Cauchy sequence is convergent.

Remark 1.4. Thanks to the inequality

$$
\left\|x_{n}-x_{m}\right\| \leq \max \left\{\left\|x_{j+1}-x_{j}\right\|: m \leq j \leq n-1\right\} \quad(n>m)
$$

a sequence $\left\{x_{n}\right\}$ is Cauchy if and only if $\left\{x_{n+1}-x_{n}\right\}$ converges to zero in a non- Archimedean space.

The most important examples of non-Archimedean spaces are $p$-adic numbers. A key property of p-adic numbers is that they do not satisfy the Archimedean axiom: for all $x, y>0$, there exists an integer $n$ such that $x<n y$.

Example 1.5. Let $p$ be a prime number. For any nonzero rational number $a=p^{r} \frac{m}{n}$ such that $m$ and $n$ are coprime to the prime number $p$, define the $p$-adic absolute value $|a|_{p}=p^{-r}$. Then || is a non-Archimedean norm on $\mathbb{Q}$. The completion of $\mathbb{Q}$ with respect to || is denoted by $\mathbb{Q}_{p}$ and is called the $p$-adic number field.

Note that if $p>3$, then $\left|2^{n}\right|=1$ in for each integer $n$.

In [1], the authors showed that if $f: Q_{p} \rightarrow \mathbb{R}$ is a continuous mapping such that for some $\varepsilon>0,|f(x+y)-f(x)-f(y)|<\varepsilon$ for all $x, y \in \mathbb{Q}_{p}$, then there exists a unique additive mapping $T: \mathbb{Q}_{p} \rightarrow \mathbb{R}$ such that $|f(x)-T(x)|<\varepsilon$ for all $x \in Q_{p}$. In [23], the authors investigated stability of some functional equations in non-Archimedean normed spaces. In this paper, we improve the strategy used in [23].

In Section 2, we apply a new technique to prove stability of the Cauchy equation in non-Archimedean normed spaces. We will exhibit some applications of our results in these spaces. In particular, we will give non-Archimedean version of Theorem 1.1. Furthermore, we give an example to show that the exact version of Theorem 1.1 is not valid in non-Archimedean normed spaces. The stability of the Jensen equation in non-Archimedean spaces is studied in Section 3.

Rätz in [22] considered the stability of an inequality associated to Jordan-von Neumann. The result of Rätz is improved by Fechner [5]. In [2], the authors studied the generalized Hyers-Ulam stability of functional inequalities

$$
\begin{aligned}
\left\|f\left(\frac{x-y}{2}-z\right)+f(y)+2 f(z)\right\| & \leq\left\|f\left(\frac{x+y}{2}+z\right)\right\|, \\
\|f(x)+f(y)+2 f(z)\| & \leq\left\|f\left(\frac{x+y}{2}+z\right)\right\| .
\end{aligned}
$$

Moreover, they have shown that if a function $f: X \rightarrow Y$ satisfies one of the above inequalities, then $f$ is additive. In Section 3, we will give an example to show that this result is not valid in non-Archimedean normed spaces. We will also consider the stability of these inequalities. More precisely, we will show that for suitable function $\varphi$, each function $f$ from a non-Archimedean linear 
space $X$ to a complete non-Archimedean normed space $Y$ which satisfies one of the following inequalities

$$
\begin{array}{r}
\left\|f\left(\frac{x-y}{2}-z\right)+f(y)+2 f(z)\right\| \leq \max \left\{\left\|f\left(\frac{x+y}{2}+z\right)\right\|, \varphi(x, y, z)\right\}, \\
\|f(x)+f(y)+2 f(z)\| \leq \max \left\{\left\|f\left(\frac{x+y}{2}+z\right)\right\|, \varphi(x, y, z)\right\}
\end{array}
$$

can be approximated by a unique additive mapping $T$ from $X$ to $Y$.

\section{Stability of Cauchy functional equation}

Hereafter, we will assume that $X$ linear space over a non-Archimedean field $\mathbb{K}$ with a valuation $|\cdot|$ and $Y$ is a complete non-Archimedean normed space over $\mathbb{K}$.

Let $f: X \rightarrow Y$ satisfy the functional equation

$$
f(x+y)=f(x)+f(y) \quad(x, y \in X),
$$

we call it a Cauchy equation. If for some function $\varphi: X \times X \rightarrow[0, \infty)$, the mapping $f: X \rightarrow Y$ satisfies

$$
\|f(x+y)-f(x)-f(y)\| \leq \varphi(x, y)
$$

for all $x, y \in X$, then $f$ is called a $\varphi$-approximately Cauchy function.

Theorem 2.1. Let $f: X \rightarrow Y$ be a $\varphi$-approximately Cauchy function. If for some natural number $k \in \mathbb{K}$,

$$
\lim _{n \rightarrow \infty}|k|^{n} \varphi\left(k^{-n} x, k^{-n} y\right)=0,
$$

for each $x, y \in X$. Then there exists a unique additive mapping $T: X \rightarrow Y$ such that

$$
\|f(x)-T(x)\| \leq \max \left\{|k|^{i-1} \psi\left(k^{-i} x\right): i \geq 1\right\}
$$

for all $x \in X$, where

$$
\psi(x)=\max \{\varphi(x, x), \varphi(x, 2 x), \ldots, \varphi(x,(k-1) x)\} \quad(x \in X) .
$$

Proof. By induction on $j$, we will show that for each $x \in X$ and $j \geq 2$,

$$
\|f(j x)-j f(x)\| \leq \max \{\varphi(x, x), \ldots, \varphi(x,(j-1) x)\} .
$$

Put $x=y$ in $(2.1)$ to obtain

$$
\|f(2 x)-2 f(x)\| \leq \varphi(x, x), \quad(x \in X) .
$$

This proves (2.4) for $j=2$. Let (2.4) hold for some $j>2$. Replacing $y$ by $j x$ in $(2.1)$, we see that

$$
\|f((j+1) x)-f(x)-f(j x)\| \leq \varphi(x, j x) \quad(x \in X) .
$$

Since

$$
f((j+1) x)-(j+1) f(x)=f((j+1) x)-f(x)-f(j x)+f(j x)-j f(x)
$$


for each $x \in X$, it follows from (2.6) and our induction hypothesis that (2.4) holds for all $j \geq 2$. In particular

$$
\|f(k x)-k f(x)\| \leq \psi(x) \quad(x \in X) .
$$

Replacing $x$ by $k^{-n} x$ in (2.7), by the inequality (2.2), we see that

$$
\left.\left\|f\left(k^{-(n-1)} x\right)-k f\left(k^{-n} x\right)\right\|\right) \leq \psi\left(k^{-n} x\right) \quad(x \in X, n=0,1,2, \ldots) .
$$

By multiplying both sides of the above inequality in $|k|^{n-1}$, we get to (2.9)

$$
\left\|k^{n-1} f\left(k^{-(n-1)} x\right)-k^{n} f\left(k^{-n} x\right)\right\| \leq|k|^{n-1} \psi\left(k^{-n} x\right) \quad(x \in X, n=0,1,2, \ldots) .
$$

By Remark 1.4 and the fact that $\lim _{n \rightarrow \infty}|k|^{n} \psi\left(k^{-n} x\right)=0$, the inequality (2.9) shows that $\left\{k^{n} f\left(k^{-n} x\right)\right\}$ is a Cauchy sequence in the complete nonArchimedean space $Y$.

Let $T(x)=\lim _{n \rightarrow \infty} k^{n} f\left(k^{-n} x\right)$. Since for each $n \geq 1$ and $x \in X$,

$$
\begin{aligned}
\left\|f(x)-k^{n} f\left(k^{-n} x\right)\right\| & =\left\|\sum_{i=1}^{n} k^{i-1} f\left(k^{-(i-1)} x\right)-k^{i} f\left(k^{-i} x\right)\right\| \\
& \leq \max \left\{\left\|k^{i-1} f\left(k^{-(i-1)} x\right)-k^{i} f\left(k^{-i} x\right)\right\| ; 1 \leq i \leq n\right\} \\
& \leq \max \left\{|k|^{i-1} \psi\left(k^{-i} x\right): 1 \leq i \leq n\right\},
\end{aligned}
$$

by taking limit as $n \rightarrow \infty$ of both sides of this inequality we see that

$$
\|f(x)-T(x)\| \leq \max \left\{|k|^{i-1} \psi\left(k^{-i} x\right): i \geq 1\right\} \quad(x \in X) .
$$

Note that the maximum of the right hand side exists, since $\lim _{n \rightarrow \infty}|k|^{n} \psi\left(k^{n} x\right)=$ 0 . This proves (2.3). It follows from (2.1), (2.2) and (2.3) that for all $x, y \in X$,

$$
\begin{aligned}
& \|T(x+y)-T(x)-T(y)\| \\
= & \lim _{n \rightarrow \infty}\left\|k^{n} f\left(k^{-n}(x+y)\right)-k^{n} f\left(k^{-n} x\right)-k^{n} f\left(k^{-n} y\right)\right\| \\
\leq & \lim _{n \rightarrow \infty}|k|^{n} \varphi\left(k^{-n} x, k^{-n} y\right)=0 .
\end{aligned}
$$

Hence $T$ is additive. Now if $T^{\prime}: X \rightarrow Y$ is another additive map such that

$$
\left\|T^{\prime}(x)-f(x)\right\| \leq \max \left\{|k|^{i-1} \psi\left(k^{-i} x\right): i \geq 1\right\}
$$

for all $x \in X$, then for each $n \in \mathbb{N}, x \in X$ we have

$$
\begin{aligned}
\left\|T(x)-T^{\prime}(x)\right\| & =\left\|k^{n} T\left(k^{-n} x\right)-k^{n} T^{\prime}\left(k^{-n} x\right)\right\| \\
& \leq|k|^{n} \max \left\{\| T\left(k^{-n} x-f\left(k^{-n} x\right)\|,\| f\left(k^{-n} x-T^{\prime}\left(k^{-n} x\right) \|\right\}\right.\right. \\
& \leq \max \left\{|k|^{n+j} \psi\left(k^{-(n+j-1)} x\right): j \geq 1\right\} \\
& =\max \left\{|k|^{j-1} \psi\left(k^{-j} x\right) ; j \geq n\right\} .
\end{aligned}
$$

By our assumption, the last term of the above inequality tends to zero as $n$ tends to infinity. Hence $T=T^{\prime}$. 
The following result can be regard as non-Archimedean version of Theorem 1.1.

Corollary 2.2. Let $X$ and $Y$ be non-Archimedean normed spaces over $\mathbb{K}$. If $Y$ is complete and for some $0 \leq r<1, f: X \rightarrow Y$ satisfies the condition

$$
\|f(x+y)-f(x)-f(y)\| \leq \theta\left(\|x\|^{r}+\|y\|^{r}\right) \quad \forall x, y \in X .
$$

Then there exists a unique additive mapping $T: X \rightarrow Y$ such that

$$
\|f(x)-T(x)\| \leq 2 \theta|k|^{-r}\|x\|^{r},
$$

where $k$ is the first integer such that $|k|<1$, we will assume that such an element exists.

Proof. Let $\varphi(x, y)=\theta\left(\|x\|^{r}+\|y\|^{r}\right)$. Since $|k|<1$ and $1-r>0$,

$$
\lim _{n \rightarrow \infty}|k|^{n} \varphi\left(k^{-n} x, k^{-n} y\right)=\lim _{n \rightarrow \infty}|k|^{n(1-r)} \varphi(x, y)=0 \quad(x \in X) .
$$

Therefore the conditions of Theorem 2.1 are satisfied. It is easy to see that

$$
\psi(x)=\max \{\varphi(x, x), \ldots, \varphi(x,(k-1) x)\}=2 \theta\|x\|^{r} \quad(x \in X)
$$

and

$$
\max \left\{|k|^{n-1} \psi\left(k^{-n} x\right): n \geq 1\right\}=2 \theta|k|^{-r}\|x\|^{r} \quad(x \in X) .
$$

By Theorem 2.1 there is a unique additive mapping $T: X \rightarrow Y$ such that (2.10) holds.

However, the following example shows that the same result of Theorem 1.1 is not true in non-Archimedean normed spaces.

Example 2.3. Let $p>2$ and $f: \mathbb{Q}_{p} \rightarrow \mathbb{Q}_{p}$ be defined by $f(x)=2$,. Then for $\varepsilon=1$,

$$
|f(x+y)-f(x)-f(y)|=1 \leq \varepsilon \quad\left(x, y \in \mathbb{Q}_{p}\right) .
$$

However neither $\left\{2^{-n} f\left(2^{n} x\right)\right\}$ nor $\left\{2^{n} f\left(2^{-n} x\right)\right\}$ is a Cauchy sequence. In fact, by using the fact that $|2|=1$, we have

$$
\left|2^{-n} f\left(2^{n} x\right)-2^{-(n+1)} f\left(2^{(n+1)} x\right)\right|=\left|2^{-n} .2-2^{-(n+1)} .2\right|=\left|2^{-n}\right|=1
$$

and

$$
\left|2^{n} f\left(2^{-n} x\right)-2^{n+1} f\left(2^{-(n+1)} x\right)\right|=\left|2^{n} \cdot 2-2^{n+1} \cdot 2\right|=\left|2^{n+1}\right|=1
$$

for all $x \in \mathbb{Q}_{p}$ and $n \in \mathbb{N}$. Hence these sequences are not convergent in $\mathbb{Q}_{p}$.

Let $f, g, h: X \rightarrow Y$ satisfy the functional equation

$$
f(x+y)=g(x)+h(y)
$$

for all $x, y \in X$, we call it a Pexider Cauchy equation. In the next result, we discuss about the stability of Pexider Cauchy equation in non-Archimedean spaces. 
Theorem 2.4. Let $f, g, h: X \rightarrow Y$ and $\varphi: X \times X \rightarrow[0, \infty)$ satisfy the inequality

$$
\|f(x+y)-g(x)-h(y)\| \leq \varphi(x, y) \quad(x, y \in X) .
$$

and (2.2) holds for some integer $k \in \mathbb{K}$, then there exists a unique additive map $T: X \rightarrow Y$ such that for all $x \in X$

$$
\|f(x)-f(0)-T(x)\| \leq \max \left\{|k|^{n-1} \psi\left(k^{-n} x\right): n \geq 1\right\}
$$

$$
\|g(x)-g(0)-T(x)\| \leq \max \left\{\varphi(x, 0), \varphi(0,0),|k|^{n-1} \psi\left(k^{-n} x\right): n \geq 1\right\}
$$

where

$$
\begin{aligned}
\psi(x)=\max \{ & \varphi(x, x), \ldots, \varphi(x,(k-1) x), \\
& \varphi(0, x), \ldots, \varphi(0,(k-1) x), \\
& \varphi(x, 0), \ldots, \varphi((k-1) x, 0), \varphi(0,0)\}
\end{aligned}
$$

for each $x \in X$.

Proof. Define $f_{1}=f-f(0), g_{1}=g-g(0), h_{1}=h-h(0)$, then by $(2.11)$

$$
\left\|f_{1}(x+y)-g_{1}(x)-h_{1}(y)\right\| \leq \max \{\varphi(x, y), \varphi(0,0)\} \quad(x, y \in X) .
$$

Put $y=0$ in (2.15) to obtain

$$
\left\|f_{1}(x)-g_{1}(x)\right\| \leq \max \{\varphi(x, 0), \varphi(0,0)\} \quad(x \in X) .
$$

Letting $x=0$ in (2.15), we see that

$$
\left\|f_{1}(y)-h_{1}(y)\right\| \leq \max \{\varphi(0, y), \varphi(0,0)\} \quad(y \in X) .
$$

It follows from (2.15), (2.16) and (2.17) that for each $x, y \in X$,

$$
\left\|f_{1}(x+y)-f_{1}(x)-f_{1}(y)\right\| \leq \max \{\varphi(0,0), \varphi(x, y), \varphi(x, 0), \varphi(0, y)\} .
$$

Put

$$
\varphi_{1}(x, y)=\max \{\varphi(0,0), \varphi(x, y), \varphi(x, 0), \varphi(0, y)\} \quad(x, y \in X) .
$$

Then $\lim _{n \rightarrow \infty}|k|^{n} \varphi_{1}\left(k^{-n} x, k^{-n} y\right)=0$. Thanks to Theorem 2.1, there exists a unique additive mapping $T: X \rightarrow Y$ which satisfies the inequality (2.12).

By (2.12) and (2.16), we see that for each $x \in X$

$$
\begin{aligned}
\|g(x)-g(0)-T(x)\| & \leq \max \left\{\left\|g_{1}(x)-f_{1}(x)\right\|,\left\|f_{1}(x)-T(x)\right\|\right\} \\
& \leq \max \left\{\varphi(x, 0), \varphi(0,0),|k|^{n-1} \psi\left(k^{-n} x\right): n \geq 1\right\}
\end{aligned}
$$

which proves (2.13). It follows from (2.12) and (2.17) that for each $x \in X$,

$$
\begin{aligned}
\|h(x)-h(0)-T(x)\| & \leq \max \left\{\left\|h_{1}(x)-f_{1}(x)\right\|,\left\|f_{1}(x)-T(x)\right\|\right\} \\
& \leq \max \left\{\varphi(0, x), \varphi(0,0),|k|^{n-1} \psi\left(k^{-n} x\right): n \geq 1\right\}
\end{aligned}
$$


hence (2.14) holds.

Corollary 2.5. Let $f, g, h: X \rightarrow Y$ satisfy

$$
\|f(x+y)-g(x)-h(y)\| \leq \theta\left(\|x\|^{r}\|y\|^{s}\right) \quad(\forall x, y \in X)
$$

for some fixed $\theta>0$ and $r, s \geq 0$ with $r+s<1$. Let $\mathbb{K}$ contains positive integers with valuation strictly less than one and $k \in \mathbb{K}$ be the first integer with this property. Then there exists a unique additive mapping $T: X \rightarrow Y$ such that for each $x \in X$,

$$
\begin{aligned}
& \|f(x)-f(0)-T(x)\| \leq \theta|k|^{-r-s}\|x\|^{r+s}, \\
& \|g(x)-g(0)-T(x)\| \leq \theta|k|^{-r-s}\|x\|^{r+s}, \\
& \|h(x)-h(0)-T(x)\| \leq \theta|k|^{-r-s}\|x\|^{r+s} .
\end{aligned}
$$

Proof. Let $\varphi(x, y)=\theta\left(\|x\|^{r}\|y\|^{s}\right)$. Then for each $x, y \in X$, we have

$$
\lim _{n \rightarrow \infty}|k|^{n} \varphi\left(k^{-n} x, k^{-n} y\right)=\lim _{n \rightarrow \infty} \theta|k|^{n(1-r-s)}\|x\|^{r}\|y\|^{s}=0 .
$$

Therefore the conditions of Theorem 2.4 are fulfilled. By Theorem 2.4 and straightforward computation one can find a unique additive mapping $T: X \rightarrow$ $Y$ such that (2.12), (2.13) and (2.14) hold.

\section{Hyers-Ulam-Rassias stability for Jensen equation}

We start this section by considering the stability of the Jensen equation

$$
2 f\left(\frac{x+y}{2}\right)=f(x)+f(y)
$$

where $f$ is a mapping from a vector spaces $X$ to a non-Archimedean space $Y$. Later, we will show that under some conditions on $X$ and $\varphi: X \times X \times X \rightarrow$ $[0, \infty)$ each function $f: X \rightarrow Y$ which satisfies one of the inequalities

$$
\begin{array}{r}
\left\|f\left(\frac{x-y}{2}-z\right)+f(y)+2 f(z)\right\| \leq \max \left\{\left\|f\left(\frac{x+y}{2}+z\right)\right\|, \varphi(x, y, z)\right\} \\
\|f(x)+f(y)+2 f(z)\| \leq \max \left\{\left\|f\left(\frac{x+y}{2}+z\right)\right\|, \varphi(x, y, z)\right\}
\end{array}
$$

can be approximated by a suitable additive mapping from $X$ to $Y$.

Theorem 3.1. Let $\varphi: X \times X \rightarrow[0, \infty)$ and $f: X \rightarrow Y$ satisfy

$$
\left\|2 f\left(\frac{x+y}{2}\right)-f(x)-f(y)\right\| \leq \varphi(x, y) \quad(x, y \in X) .
$$

If for some natural number $k \in \mathbb{K}$

$$
\lim _{n \rightarrow \infty}|k|^{n} \varphi\left(k^{-n} x, k^{-n} y\right)=0
$$

then there exists a unique additive mapping $T: X \rightarrow Y$ such that

$$
\|f(x)-f(0)-T(x)\| \leq \max \left\{|k|^{i-1} \psi\left(k^{-i} x\right): i \geq 1\right\}
$$


for all $x \in X$, where

$$
\begin{gathered}
\psi(x)=\max \{\varphi(x, x), \varphi(x, 2 x), \ldots, \varphi(x,(k-1) x), \\
\varphi(2 x, 0), \varphi(3 x, 0), \ldots, \varphi(k x, 0)\}
\end{gathered}
$$

for all $x \in X$.

Proof. Let $f_{1}(x)=f(x)-f(0)$ for all $x \in X$. By (3.1),

$$
\left\|2 f_{1}\left(\frac{x+y}{2}\right)-f_{1}(x)-f_{1}(y)\right\| \leq \varphi(x, y) \quad(x, y \in X) .
$$

Replacing $x$ by $x+y$ and $y$ by 0 in (3.3), we see that

$$
\left.\left\|2 f_{1}\left(\frac{x+y}{2}\right)-f_{1}(x+y)\right\| \leq \varphi(x+y, 0)\right) \quad(x, y \in X) .
$$

It follows from (3.3) and the above inequality that

(3.4) $\left\|f_{1}(x+y)-f_{1}(x)-f_{1}(y)\right\| \leq \max \{\varphi(x, y), \varphi(x+y, 0)\} \quad(x, y \in X)$.

Let $\varphi_{1}(x, y)=\max \{\varphi(x, y), \varphi(x+y, 0)\}$. Then by (3.4),

$$
\left\|f_{1}(x+y)-f_{1}(x)-f_{1}(y)\right\| \leq \varphi_{1}(x, y) \quad(x, y \in X) .
$$

By Theorem 2.1, we can find a unique additive mapping $T: X \rightarrow Y$ such that

$$
\begin{aligned}
\|f(x)-f(0)-T(x)\| & =\left\|f_{1}(x)-T(x)\right\| \\
& \leq \max \left\{|k|^{i-1} \psi\left(k^{-i} x\right): i \geq 1\right\} \quad(x \in X) .
\end{aligned}
$$

Let $f, g, h: X \rightarrow Y$ satisfy the functional equation

$$
2 f\left(\frac{x+y}{2}\right)=g(x)+h(y)
$$

for all $x, y \in X$, we call it a Pexider Jensen equation.

The following example shows an application of Theorem 3.1 for stability of a Pexider Jensen equation.

Example 3.2. Let $p>2$ be a prime number, let $X$ and $Y$ be non-Archimedean normed spaces over $\mathbb{Q}_{p}$ and $Y$ is complete. If $f, g, h: X \rightarrow Y$ satisfy the inequality

$$
\left\|2 f\left(\frac{x+y}{2}\right)-g(x)-h(y)\right\| \leq \theta \max \left\{\|x\|^{r},\|y\|^{r}\right\} \quad(x, y \in X)
$$

for some $\theta>0,0<r<1$ and all $x, y \in X$. Then there exists a unique additive mapping $T: X \rightarrow Y$ such that for all $x \in X$,

$$
\begin{aligned}
& \|f(x)-f(0)-T(x)\| \leq \theta p^{r}\|x\|^{r}, \\
& \|g(x)-g(0)-T(x)\| \leq \theta p^{r}\|x\|^{r}, \\
& \|h(x)-h(0)-T(x)\| \leq \theta p^{r}\|x\|^{r} .
\end{aligned}
$$


To prove this, let $f_{1}=f-f(0), g_{1}=g-g(0)$ and $h_{1}=h-h(0)$, then

$$
\left\|2 f_{1}\left(\frac{x+y}{2}\right)-g_{1}(x)-h_{1}(y)\right\| \leq \theta \max \left\{\|x\|^{r},\|y\|^{r}\right\} \quad(x, y \in X) .
$$

Put $y=x$ in (3.10) to obtain

$$
\begin{aligned}
\left\|2 f_{1}(x)-g_{1}(x)-h_{1}(x)\right\| & \leq \theta\|x\|^{r}, \\
\left\|2 f_{1}(y)-g_{1}(y)-h_{1}(y)\right\| & \leq \theta\|y\|^{r} .
\end{aligned}
$$

By changing the rule of $x$ and $y$ in (3.10), we see that

$$
\left\|2 f_{1}\left(\frac{x+y}{2}\right)-g_{1}(y)-h_{1}(x)\right\| \leq \theta \max \left\{\|x\|^{r},\|y\|^{r}\right\} \quad(x, y \in X) .
$$

Since $|2|=1$, it follows from (3.10), (3.11), (3.12) and (3.13) that

$$
\left\|2 f_{1}\left(\frac{x+y}{2}\right)-f_{1}(y)-f_{1}(x)\right\| \leq \theta \max \left\{\|x\|^{r},\|y\|^{r}\right\} \quad(x, y \in X) .
$$

In Theorem 3.1, let $k=p$ and $\varphi(x, y)=\theta \max \left\{\|x\|^{r},\|y\|^{r}\right\}$. Then

$$
\lim _{n \rightarrow \infty}|p|^{n} \varphi\left(p^{-n} x, p^{-n} y\right)=\lim _{n \rightarrow \infty} \frac{\theta}{p^{n(1-r)}} \max \left\{\|x\|^{r},\|y\|^{r}\right\}=0 \quad(x, y \in X) .
$$

By Theorem 3.1, there is a unique additive mapping $T: X \rightarrow Y$ such that

$$
\|f(x)-f(0)-T(x)\| \leq \max \left\{|p|^{n-1} \psi\left(p^{-n} x\right): n \geq 1\right\} \quad(x \in X) .
$$

It is easy to see that in this case $\psi(x)=\theta\|x\|^{r}$, hence (3.7) holds. Put $y=0$ in (3.10) to obtain

$$
\left\|2 f_{1}\left(\frac{x}{2}\right)-g_{1}(x)\right\| \leq \theta\|x\|^{r} \quad(x \in X) .
$$

It follows from (3.7) and (3.15) that

$$
\begin{aligned}
& \|g(x)-g(0)-T(x)\| \\
\leq & \max \left\{\left\|g_{1}(x)-2 f_{1}\left(\frac{x}{2}\right)\right\|,\left\|2 f_{1}\left(\frac{x}{2}\right)-2 T\left(\frac{x}{2}\right)\right\|\right\} \quad(x \in X) \\
\leq & \max \left\{\theta\|x\|^{r}, \theta p^{r}\|x\|^{r}\right\}=\theta p^{r}\|x\|^{r} .
\end{aligned}
$$

This proves (3.8). Similar argument shows that (3.9) holds.

In [2], the authors investigated the stability of inequalities related to Jordanvon Neumann-type Jensen additive functional equations [22].

In the next results, we consider the following similar inequalities

$$
\begin{aligned}
\left\|f\left(\frac{x-y}{2}-z\right)+f(y)+2 f(z)\right\| & \leq \max \left\{\left\|f\left(\frac{x+y}{2}+z\right)\right\|, \varphi(x, y, z)\right\}, \\
\| 3.17) \quad\|f(x)+f(y)+2 f(z)\| & \leq \max \left\{\left\|f\left(\frac{x+y}{2}+z\right)\right\|, \varphi(x, y, z)\right\}
\end{aligned}
$$

in non-Archimedean normed linear spaces. 
Theorem 3.3. Let $\varphi: X \times X \times X \rightarrow[0, \infty)$ and $f: X \rightarrow Y$ satisfy (3.16). If for some natural number $k \in \mathbb{K}$ with $|k|<1$

$$
\lim _{n \rightarrow \infty}|k|^{n} \varphi\left(k^{-n} x, k^{-n} y, k^{-n} z\right)=0 \quad(x, y, z \in X),
$$

then there exists a unique additive mapping $T: X \rightarrow Y$ such that

$$
\|f(x)-f(0)-T(x)\| \leq \max \left\{|k|^{i-1} \psi\left(k^{-i} x\right): i \geq 1\right\}
$$

for all $x \in X$, where

$$
\begin{array}{r}
\psi(x)=\max \left\{\varphi_{1}(x, x), \varphi_{1}(x, 2 x), \ldots, \varphi_{1}(x,(k-1) x),\right. \\
\left.\varphi_{1}(2 x, 0), \varphi_{1}(3 x, 0), \ldots, \varphi(k x, 0)\right\}
\end{array}
$$

and

$$
\begin{array}{r}
\varphi_{1}(x, y)=\max \left\{\|f(0)\|, \varphi(x, x,-x), \varphi\left(x, y, \frac{-x-y}{2}\right),\right. \\
\left.\varphi\left(\frac{x+y}{2}, \frac{x+y}{2}, \frac{-x-y}{2}\right)\right\}
\end{array}
$$

for all $x \in X$.

Proof. Put $y=x$ and $z=-x$ in (3.16) to obtain

$$
\|2 f(x)+2 f(-x)\| \leq \max \{\|f(0)\|, \varphi(x, x,-x)\} \quad(x \in X) .
$$

Replacing $z$ by $\frac{-x-y}{2}$ in $(3.16)$, we get

$$
\left\|f(x)+f(y)+2 f\left(\frac{-x-y}{2}\right)\right\| \leq \max \left\{\|f(0)\|, \varphi\left(x, y, \frac{-x-y}{2}\right)\right\}
$$

for all $x, y \in X$. By (3.19) and (3.20) for all $x, y \in X$, we have

$$
\begin{aligned}
& \left\|2 f\left(\frac{x+y}{2}\right)-f(x)-f(y)\right\| \\
\leq & \max \left\{\|f(0)\|, \varphi\left(x, y, \frac{-x-y}{2}\right), \varphi\left(\frac{x+y}{2}, \frac{x+y}{2}, \frac{-x-y}{2}\right)\right\} .
\end{aligned}
$$

Since $|k|<1, \lim _{n \rightarrow \infty}|k|^{n}|| \varphi(0) \|=0$. Therefore by (3.18),

$$
\lim _{n \rightarrow \infty}|k|^{n} \varphi_{1}\left(k^{-n} x, k^{-n} y\right)=0 .
$$

Thanks to Theorem 3.1, we get to the desire result.

Remark 3.4. In [2] it is shown that if a function $f$ satisfies the inequality

$$
\left\|f\left(\frac{x-y}{2}-z\right)+f(y)+2 f(z)\right\| \leq\left\|f\left(\frac{x+y}{2}+z\right)\right\|,
$$

then $f$ is additive. Note that this result is not valid for non-Archimedean normed spaces. To see this define $f: \mathbb{Q}_{p} \rightarrow \mathbb{Q}_{p}$ for prime number $p>2$, by $f(x)=4$, then

$$
\left|f\left(\frac{x-y}{2}-z\right)+f(y)+f(z)\right|=1=\left|f\left(\frac{x+y}{2}+z\right)\right| \quad\left(x, y, z \in \mathbb{Q}_{p}\right) .
$$


However $f$ is not additive.

Theorem 3.5. Let $\varphi: X \times X \times X \rightarrow[0, \infty)$ and $f: X \rightarrow Y$ satisfy (3.17). If for some natural number $k \in \mathbb{K}$ with $|k|<1$,

$$
\lim _{n \rightarrow \infty}|k|^{n} \varphi\left(k^{-n} x, k^{-n} y, k^{-n} z\right)=0 \quad(x, y, z \in X),
$$

then there exists a unique additive mapping $T: X \rightarrow Y$ such that

$$
\|f(x)-f(0)-T(x)\| \leq \max \left\{|k|^{i-1} \psi\left(k^{-i} x\right): i \geq 1\right\}
$$

for all $x \in X$, where

$$
\begin{gathered}
\psi(x)=\max \left\{\varphi_{1}(x, x), \varphi_{1}(x, 2 x), \ldots, \varphi_{1}(x,(k-1) x),\right. \\
\left.\varphi_{1}(2 x, 0), \varphi_{1}(3 x, 0), \ldots, \varphi(k x, 0)\right\}
\end{gathered}
$$

and

$$
\varphi_{1}(x, y)=\max \left\{\|2 f(0)\|, \varphi\left(x, y, \frac{-x-y}{2}\right), \varphi\left(\frac{x+y}{2}, \frac{x+y}{2}, \frac{x+y}{2}\right)\right\}
$$

for all $x \in X$.

Proof. Let $z=\frac{-x-y}{2}$ in (3.17) to obtain

$$
\begin{aligned}
& \left\|f(x)+f(y)+2 f\left(\frac{-x-y}{2}\right)\right\| \\
\leq & \max \left\{\|2 f(0)\|, \varphi\left(x, y, \frac{-x-y}{2}\right)\right\} \quad(x, y \in X) .
\end{aligned}
$$

Replacing $x, y$ by $\frac{x+y}{2}$ and $z$ by $\frac{-x-y}{2}$ in (3.17), we get

$$
\left\|2 f\left(\frac{x+y}{2}\right)+2 f\left(\frac{-x-y}{2}\right)\right\| \leq \max \left\{\|2 f(0)\|, \varphi\left(\frac{x+y}{2}, \frac{x+y}{2}, \frac{-x-y}{2}\right)\right\}
$$

for all $x, y \in X$. By (3.23) and (3.24) for all $x, y \in X$, we have

$$
\begin{aligned}
& \left\|2 f\left(\frac{x+y}{2}\right)-f(x)-f(y)\right\| \\
\leq & \max \left\{\|2 f(0)\|, \varphi\left(x, y, \frac{-x-y}{2}\right), \varphi\left(\frac{x+y}{2}, \frac{x+y}{2}, \frac{-x-y}{2}\right)\right\} .
\end{aligned}
$$

Similar argument used in the end of Theorem 3.3 can be used to prove the result.

Example 3.6. Let $X$ and $Y$ are normed linear space over $\mathbb{Q}_{p}$, where $p>2$ is a prime number. If $Y$ is complete and $f: X \rightarrow Y$ satisfies the condition

$$
\|f(x)+f(y)+2 f(z)\| \leq \max \left\{\left\|f\left(\frac{x+y}{2}+z\right)\right\|, \varepsilon\right\}
$$

for each $x, y, z$ in $X$, then (3.17) for $\varphi(x, y, z)=\varepsilon$ holds. Since

$$
\lim _{n \rightarrow \infty}|p|^{n} \varphi\left(k^{-n} x, k^{-n} y, k^{-n} z\right)=\lim _{n \rightarrow \infty} \frac{\varepsilon}{p^{n}}=0
$$


for all $x, y, z \in X$ and

$$
\psi(x)=\max \{|2| \| f(0)||, \varepsilon\}=\max \{\|f(0) \mid\|, \varepsilon\}
$$

for all $x \in X$. Straightforward computation, using Theorem 3.5, show that there is a unique additive mapping $T: X \rightarrow Y$ such that

$$
\|f(x)-f(0)-T(x)\| \leq \frac{\max \{\|f(0)\|, \varepsilon\}}{p} \quad(x \in X) .
$$

\section{References}

[1] L. M. Arriola and W. A. Beyer, Stability of the Cauchy functional equation over p-adic fields, Real Anal. Exchange 31 (2005/06), no. 1, 125-132.

[2] Y. S. Cho and H. M. Kim, Stability of functional inequalities with Cauchy-Jensen additive mappings, Abstr. Appl. Anal. (2007), Art. ID 89180, 13 pp.

[3] S. Czerwik, Functional Equations and Inequalities in Several Variables, World Scientific, River Edge, NJ, 2002.

[4] D. Deses, On the representation of non-Archimedean objects, Topology Appl. 153 (2005), no. 5-6, 774-785.

[5] W. Fechner, Stability of a functional inequality associated with the Jordan-von Neumann functional equation, Aequationes Math. 71 (2006), no. 1-2, 149-161.

[6] P. Găvruta, A generalization of the Hyers-Ulam-Rassias stability of approximately additive mappings, J. Math. Anal. Appl. 184 (1994), no. 3, 431-436.

[7] K. Hensel, Uber eine news Begrundung der Theorie der algebraischen Zahlen, Jahresber. Deutsch. Math. Verein 6 (1897), 83-88.

[8] D. H. Hyers, On the stability of the linear functional equation, Proc. Nat. Acad. Sci. U.S.A. 27 (1941), 222-224.

[9] D. H. Hyers, G. Isac, and Th. M. Rassias, Stability of Functional Equations in Several Variables, Birkhäuser, Basel, 1998.

[10] K. Jun and H. Kim, On the Hyers-Ulam-Rassias stability problem for approximately $k$-additive mappings and functional inequalities, Math. Inequal. Appl. 10 (2007), no. 4, 895-908.

[11] S.-M. Jung, Hyers-Ulam-Rassias Stability of Functional Equations in Mathematical Analysis, Hadronic Press, Palm Harbor, 2001.

[12] _ Hyers-Ulam-Rassias stability of Jensen's equation and its application, Proc. Amer. Math. Soc. 126 (1998), no. 11, 3137-3143.

[13] A. K. Katsaras and A. Beoyiannis, Tensor products of non-Archimedean weighted spaces of continuous functions, Georgian Math. J. 6 (1999), no. 1, 33-44.

[14] A. Khrennikov, Non-Archimedean Analysis: quantum paradoxes, dynamical systems and biological models, Mathematics and its Applications, 427. Kluwer Academic Publishers, Dordrecht, 1997.

[15] Z. Kominek, On a local stability of the Jensen functional equation, Demonstratio Math. 22 (1989), no. 2, 499-507.

[16] L. Li, J. Chung, and D. Kim, Stability of Jensen equations in the space of generalized functions, J. Math. Anal. Appl. 299 (2004), no. 2, 578-586.

[17] A. K. Mirmostafaee, M. Mirzavaziri, and M. S. Moslehian, Fuzzy stability of the Jensen functional equation, Fuzzy Sets and Systems 159 (2008), no. 6, 730-738.

[18] A. K. Mirmostafaee and M. S. Moslehian, Fuzzy versions of Hyers-Ulam-Rassias theorem, Fuzzy Sets and Systems 159 (2008), no. 6, 720-729.

[19] P. J. Nyikos, On some non-Archimedean spaces of Alexandrof and Urysohn, Topology Appl. 91 (1999), 1-23. 
[20] J. C. Parnami and H. L. Vasudeva, On Jensen's functional equation, Aequationes Math. 43 (1992), no. 2-3, 211-218.

[21] Th. M. Rassias, On the stability of the linear mapping in Banach spaces, Proc. Amer. Math. Soc. 72 (1978), no. 2, 297-300.

[22] J. Rätz, On inequalities associated with the Jordan-von Neumann functional equation, Aequationes Math. 66 (2003), no. 1-2, 191-200.

[23] M. Sal Moslehian and T. M. Rassias, Stability of functional equations in nonArchimedean spaces, Appl. Anal. Discrete Math. 1 (2007), no. 2, 325-334.

[24] S. M. Ulam, Problems in Modern Mathematics, Science Editions, John Wiley \& Sons, 1964.

Department of Mathematics

School of Mathematical Sciences

Ferdowsi University of MashHad

P. O. Box 1159, MashHad, Iran

AND

Center of Excellence in Analysis on Algebraic Structures

Ferdowsi University of MashHad, Iran

E-mail address: mirmostafaei@math.um.ac.ir, mirmostafaei@ferdowsi.um.ac.ir 